\title{
Developmental Profiling - The Tool of Choice to Measure Deficit, Plan Intervention and Monitor Outcome in Children with Developmental Disabilities
}

\author{
Prathibha Karanth*, Tanya Dash and Tanushree Saxena-Chandhok
}

The Com DEALL Trust, No. 224, 6th 'A' main, 2nd Block, HRBR Layout, Bangalore- 560 043, India

\begin{abstract}
This article highlights the transition from a more traditional IQ measure to a profile based approach of assessment, in children with developmental disabilities. Clinically, developmental disorders are heterogeneous conditions, which have made uniformity of assessments across settings, centers and countries elusive. A decade ago, intelligence tests were given priority for assessing children. However, IQ scores had little direct bearing for the planning of intervention. This article highlights an assessment tool that, not only provides a quick understanding of the child's overall skill across eight essential developmental domains, but also offers guidance for intervention planning on the basis of assessment.
\end{abstract}

Keywords: Developmental disorder, IQ assessment, Profile based assessment, Early intervention, Communication DEALL Developmental Checklist (CDDC).

\section{INTRODUCTION}

'Developmental disorders' refer to disorders, which are due to impairments in physical and/or mental capabilities, resulting in overall developmental difficulties. The difficulties may include inadequacies in several areas such as, perceptual, motor, communication and social skills. 'Developmental disabilities' cover a range of disorders including Intellectual Disabilities (ID), Cerebral Palsy (CP), Hearing Impairment $(\mathrm{HI})$ and Autism Spectrum Disorders (ASD). These disorders typically are present in early childhood and continue to affect the individual throughout the life span. Traditional methods of assessment and intervention have undergone gradual changes with revision of various theories and practices related to developmental disabilities.

A thorough assessment of the strengths and weaknesses of the child with a developmental disability is essential to understand his/her intervention needs. Several types of assessment procedures have been developed and applied over the years. Traditionally, the assessment of intelligence was given primary importance. In the recent past there has been a greater shift towards more comprehensive assessment tools [1]. Intervention programs developed for children with developmental disabilities have also seen changes, over the years with a focus on broad based early intervention. Increasingly the importance of comprehensive assessment protocols and the need to link

*Address correspondence to this author at The Com DEALL Trust, No. 224, 6th 'A' main, 2nd Block, HRBR Layout, Bangalore- 560 043, India;

Tel: 9480334809; E-mail: communicationdeall@gmail.com assessments with intervention planning are being acknowledged [2].

The current paper focuses on issues related to assessment and intervention in developmental disabilities, with Autism Spectrum Disorders (ASD) as an example. The paper highlights the importance of the need to profile skills of every child with developmental disabilities, across several developmental domains, thus increasing the focus and scope of intervention as well as enabling effective monitoring of progress. This is in contrast to the traditional methods of assessments, where there is reliance on only one measure - the IQ.

\section{IQ AS AN ASSESSMENT MEASURE}

Intelligence Quotient (IQ) scores measured intelligence and were considered as a universal entity. Although there are numerous tests to assess IQ [3,4,5] they are culturally biased, which may include test item bias as well as construct validity bias. IQ is usually used as a measure to understand academic achievement as well as predict life outcome measures [6], which may be influenced by factors such as age of the child with developmental disability and age at which intervention was started. Alfred Binet the pioneer of intelligence testing was criticized for the use of a single score to notify intelligence (i.e. IQ).

A single score, as measured by the Intelligence Quotient (IQ), is limiting in that it cannot explain the multidimensional aspects of intelligence. Tests of intelligence usually result in IQ scores, which is a summary score on a standardized measure. IQ scores 
may reflect influence of sensory, motor and language skills. Thus a child with typical cognitive skills may show low $I Q$ as a product of impairment in sensory/motor/language skills [2]. Although, the Stanford Binet test, reduces intelligence to a single score the author himself was not convinced by the idea of expressing the complex phenomenon of intelligence as a single score [7]. He too acknowledged the likelihood of such scores negatively affecting education, livelihood and the larger sociocommunicative environment.

IQ tests ignore the intelligence required in conducting daily-life activities, social knowledge, mechanical skills and in being creative. Therefore, IQ measures are disadvantageous for use with children with disabilities, who may have better creative and mechanical skills. Over the years, criticism about the use of IQ assessments for children with developmental disabilities, when compared to other methods of assessment, have mounted. For instance, in a systematic review, spanning over six decades, a prevalence rate of $75 \%$ intellectual disability in ASD, was reported [8]. Edelson, the author of this review pointed out that the prevalence rate of intellectual disability in ASD varied as a function of the testing method used in the study. Studies using developmental and adaptive scales were able to identify intellectual disability in children with ASD better than those using only IQ tests.

Another program known as 'I laugh' criticized the use of the traditional standardized measures for children with ASD, as they lack functional utility [9]. The program, instead of relying on the estimate of IQ (i.e. lower functioning, mid-functioning and high functioning) focuses on the strength and weaknesses of the child and uses the I laugh framework's cognitive component for social relationship and academic performance across school days. Unlike IQ assessment, the focus is on more functional aspects of performance on day to day skills which are targeted in intervention.

Traditional IQ measures considered as a global measure of cognitive function, are now replaced by more broad based tests (i.e. cognitive tests and adaptive behavioural scales). Various tests of $I Q$ as well as cognitive functions result in a score derived from multiple subtests and appropriate interpretation requires understanding of the complex construction of the test. Further these tests require assessment of supplementary material for a comprehensive assessment result.
The performance on the subtasks on IQ tests requires appropriate functioning and coordination of multiple skills to achieve adequate scores. This puts children with developmental disabilities at a disadvantage, since they may have inadequate skills in specific areas or lack coordination of multiple skills. For instance, within the IQ test- the performance scale will require the integrity in performance of visual spatial abilities, fine motor coordination as well as perceptual skills. Therefore, low IQ scores can be because of fine motor limitation rather than intelligence per se.

Harris and Hengleman [10] reported that the age of the child with ASD and IQ at intake into educational programs, are crucial factors in determining the school placement of the child i.e. in a regular or special classroom. Their study suggests that there are two factors responsible for the placement of the child in a regular education system, age and IQ. Regular schooling was recommended for the children who were enrolled in an early intervention program before 48 months or had higher IQ scores (more than 80). On the other hand, special schooling was recommended for children who attended early intervention program after the age of 48 months or had lower IQ (less than 52). Restori, Katz and Lee [11] criticized the IQ/achievement discrepancy model while supporting the use of the Response to Intervention (RTI) approach for identifying children with possible learning disability for special education. They suggest that the IQ/Achievement discrepancy model hinders early identification and intervention in Specific Learning Disability (SLD). They also highlight the fact that different practitioners or school personnel may apply it differently leading to inconsistency in the identification of SLD. Thirdly, because of this discrepancy model, children with genuine educational requirement may not receive services because of the low performance on IQ measures. Thus, a slow learner may be deprived of the possibility of an appropriate special education program because of below average IQ. As an argument, the subscales of IQ may also be considered to assist intervention planning and progress estimation. However, it may not always fulfill this purpose since it mainly gives a broad indication of areas of concern rather than specific skills of the child.

Over the years, there is a greater reliance of adaptive behavioral skills assessments. Adaptive skills are demonstrated through the daily routine of the child and it is best seen by the caregiver but caregiver can also add bias to the report, where they can over or underestimate the skills of the child. Adaptive skills 
may be affected to a greater extent than cognitive skills in children with developmental disabilities [12, 13]. For example, a child with autism may have cognitive skills, which are above average, however, s/he will not be able to perform daily life skills or take care of self.

\section{IMPORTANCE OF PROFILING}

As illustrated above traditional methods such as IQ scores, unfortunately, lead to negative influence and biased judgment of children with developmental disabilities by clinicians and society. This prejudice can be avoided to an extent by profiling the individual with developmental disability. A 'profile' refers to the visual depiction of the overall skills of a child along several developmental domains. It enables the assessor to highlight areas of strength and weakness in the child with developmental disability, at a glance. Such an assessment allows the interventionist to address the immediate issues related to therapy.

Children with developmental disabilities could have various sensory, motor, and intellectual disabilities resulting in considerable variability, within and across subtypes of disability. Profiling developmental skills, help in projecting both the deficit areas and child's strengths on various tasks, irrespective of disability type and will be a more appropriate way to assess a child's performance at any given time.

Many children with developmental disability have difficulties in a wide variety of areas, such as social communication, transition from one task to another, compliance as well as motivation level toward a particular task during the assessment. It tends to be difficult to observe all the skills in an IQ test or other cognitive assessment. On the other hand, an assessment tool like the Vineland Adaptive Behavioural Scale (VABS) [14], which relies only on informants' input, can be vulnerable to biases. Therefore, there is a need to assess adaptive skills along with cognitive skills to understand the overall capabilities of a child. This understanding can easily be executed by a profile based approach.

\section{EARLY INTERVENTION AND ITS IMPORTANCE FOR CHILDREN WITH DEVELOPMENTAL DISABILITY}

Profiling is particularly of value in assessing very young children, who are difficult to test. With the recent emphasis on early intervention, which has proven long term benefits, for children with developmental disabilities [15], comprehensive early assessment too has received an impetus. The need for intervening early has been demonstrated to be valuable through a decade of research $[16,17,18]$. Positive early experiences enable imprinting on to the brain, thereby establishing stable relationships and further development [19]. Therefore, learning during the preschool years of life reaps maximum benefits and positive outcomes. As the child's developmental and educational skills improve, the dependence upon social institutions and individuals gradually decreases. This leads to vocational stability, thereby providing economic as well as social benefits, at large. Quantitative and qualitative research data [17, 20, 21] supports the notion that early intervention is effective as it enables overall long-term gains for the child and family and therefore society.

An effective intervention program relies heavily on its assessment protocol. In the case of developmental disabilities such as the ASD, diagnostic labels, assessment methods as well as intervention methodology and strategies have been ever changing. Heterogeneity seen in these populations requires a valid and reliable manner of assessment [22]. For evidence based practice too, it is important to select an appropriate method of assessment for a child with disabilities, focusing on overall, pre-linguistic skills, sensorimotor skills, language and communication, social skills, emotional skills, as well as cognitive skills. There are numerous factors influencing assessment as well as decisions for intervention such as etiology, symptoms, developmental course and outcome [22]. Effective intervention planning will be possible if the entire profile of a child is drawn and understood. Such an approach will provide a holistic understanding of the child's issues, skills and needs. Multiple accounts through direct observation as well as care-giver interview assist in describing the strengths and weaknesses of children with developmental disabilities.

\section{COMMUNICATION DEALL DEVELOPMENTAL CHECKLIST (CDDC) - A TOOL FOR PROFILING CHILDREN WITH DEVELOPMENTAL DISABILITIES}

The Communication DEALL Developmental Checklist (CDDC) [23], a tool to profile developmental skills in young children with developmental disabilities, was developed by Karanth in 2007 [23]. This quick-touse and easy screening assessment checklist measures skills for children between the ages of 0 and 6 years, across eight developmental domains. For young children the need for a quick and sensitive checklist, which would assist in effective planning for 
intervention, cannot be undermined. The CDDC can be used across a variety of developmental disabilities (ASD, Hearing Impairment, Intellectual disability etc.) and languages, to draw out a developmental profile. The CDDC, in its published version, consists of 288 questions over eight developmental domains (viz. gross motor skills, fine motor skills, activity of daily living, receptive and expressive language, cognitive skills, social skills and emotional skills). These eight domains were chosen based on the understanding of the areas affected across developmental disabilities. These domains cover the most basic areas that are typically affected across disabilities. These critical components for any intervention program are effectively inculcated in the Communication DEALL program. The Communication DEALL program [24], an intensive early intervention program for children with ASD and other developmental disabilities, addresses several areas of difficulty during the assessment process, which enable effective management planning and reflect on the child's difficulties. The Com DEALL assessment protocol- The Communication DEALL Developmental Checklist (CDDC) [23] - assesses each child along eight developmental domains and is used along with other ASD specific, assessments such as those for pre-requisite learning skills, sensoryperceptual, non-verbal and pragmatic skills, as well as behavioral issues. The CDDC profile provides a comprehensive developmental profile of the child across several domains in addition to the disorder specific measures.

The tool underwent a two-phase data collection process to standardize it [24] on 360 urban middleclass families in Bangalore, India; with 30 children (15 M and $15 \mathrm{~F}$ ) at every six month age interval, from $0-72$ months. The two-phase process of data collection included the first phase, which confirmed the soundness of the checklist (developmentally within a category and across categories) and the second phase, which completed the standardization process. An inter-rater study [25] was conducted on the CDDC, which studied correlation between ratings of raters and ratings of parental responses and also the relation between the raters and parents' responses. The results of this study showed high correlation values across all developmental domains which are greater than 0.89 . This indicated high correlation between the raters' rating, between the parental responses and between the raters' rating and parental responses. Such high correlation values suggest high reliability of the tool.
Assessments conducted with the CDDC, are carried out for each of the eight domains, separately by a group of therapists who address areas of their expertise (viz. motor domains of gross, fine motor skills and activity of daily living are addressed by physiotherapists and occupational therapists; the communication domains of receptive and expressive language area assessed by speech language therapists; and a developmental psychologist or educator assesses the cognitive, social and emotional skills). For children with suspected developmental issues, skills are assessed from the lower or basic skills to higher level skills up to the child's chronological age. For assessment, methods of direct observation and parental interviewing are used. Through the fivepoint rating, this tool allows the professional to not only draw insights about the child's current performance across different contexts but also about the possible regression of skills acquired earlier [26]. This is important for children with disabilities such as ASD. Since the CDDC collects data based on a combination of both methods of data collection (i.e. parental reports as well as observation on tasks) it is sensitive and reliable. The five- point rating scale [26] used in the tool also allows a more objective measurement and understanding of a generalized skill (i.e. skill that will be seen by informants as well as others) or one that is not generalized (i.e. a skill that is seen only by informants perhaps). The domain specific scores allow a percentage of capability to be calculated along each developmental domain and additionally across all domains. This is useful to quantify-the child's skills and obtain a gross measure of progress in intervention. The profile drawn from the scores placed on the score sheet of the checklist, allows easy planning of intervention (see Table 1).

Table 1 illustrates a five point rating scale (0-4) which has been colour coded to clearly indicate different level of performance. 0 indicates lack of skill (i.e. red color), 1 indicates regression in a particular skill (i.e. gray colour), 2 suggests inconsistency in performance (i.e. yellow colour), 3 suggests presence of skill in a particular situation only (i.e. blue colour) and 4 indicates generalized performance of a particular skill (i.e. green colour). In Table 1, rating of 3 (blue) and 1 (gray) are not present, as they were not relevant for this particular child.

The team of early interventionists' consisting of an occupational therapist or physiotherapist, speech language pathologist and educator or psychologist would initiate intervention targeting skills at baseline in 
Table 1: Developmental Profile Based on CDDC

\begin{tabular}{|c|c|c|c|c|c|c|c|c|}
\hline 12045 & & \multicolumn{7}{|c|}{ Profile of a child aged 45 months } \\
\hline Age in month & GM & FM & ADL & $\mathbf{R L}$ & EL & COG & soc & EM \\
\hline $42-48$ & 2 & 0 & 2 & 0 & 0 & 0 & 0 & 0 \\
\hline \multirow{3}{*}{$36-42$} & 4 & 4 & 4 & 0 & 0 & 0 & 2 & 0 \\
\hline & 2 & 2 & 4 & 0 & 0 & 2 & 0 & 0 \\
\hline & 4 & 2 & 4 & 2 & 0 & 0 & 0 & 0 \\
\hline $30-36$ & 4 & 2 & 4 & 4 & 2 & 0 & 0 & 0 \\
\hline \multirow[t]{2}{*}{$24-30$} & 2 & 4 & 4 & 2 & 4 & 4 & 2 & 2 \\
\hline & 2 & 4 & 4 & 4 & 0 & 4 & 0 & 0 \\
\hline \multirow{3}{*}{$18-24$} & 4 & 4 & 4 & 4 & 4 & 2 & 2 & 4 \\
\hline & 4 & 4 & 4 & 4 & 4 & 4 & 0 & 4 \\
\hline & 4 & 4 & 4 & 4 & 2 & 4 & 4 & 4 \\
\hline \multirow{3}{*}{$12-18$} & 4 & 2 & NR & 2 & 4 & 4 & 0 & 4 \\
\hline & 4 & 4 & 4 & 4 & 4 & 4 & 4 & 4 \\
\hline & 2 & 4 & 4 & 4 & 4 & 4 & 2 & 4 \\
\hline $0-6$ & 4 & 4 & 4 & 4 & 4 & 4 & 4 & 4 \\
\hline Sub-domain\% & 84.78 & 82.6 & 89.1 & 69.5 & 60.4 & 67.4 & 45.7 & 63.04 \\
\hline Domain\% & \multicolumn{3}{|c|}{85.5} & \multicolumn{2}{|c|}{65.2} & \multicolumn{3}{|c|}{58.7} \\
\hline Overall\% & \multicolumn{8}{|c|}{70.4} \\
\hline
\end{tabular}

their respective domains, moving on to higher level skills, while those at the lower level stabilize, with the goal of targeting age appropriate skills at the earliest possible time. Taking an example of young child with Autism with skill set as seen in Table 1, the interventionists were able to understand the exact level of functioning across the developmental domains.

With dedicated and intensive inputs ( 5 days a week, 3 hours a day) and planning of goals on a monthly basis, over a period of ten months, progression of the child's skill could be seen. Periodic assessments allow the measurement of improvement in skills due to therapy, over a period of time (see Table 2). Table 2 clearly indicates how the intensive intervention has measurable changes across all domains, with skills that are yet to be stabilized or generalized or achieved, seen clearly. This will assist the therapists to further plan for the child's intervention and also use the child's strengths to motivate him through the sessions.

When using the checklist across the variety of developmental disabilities, the assessor must address core assessment areas prior to using the same for a 


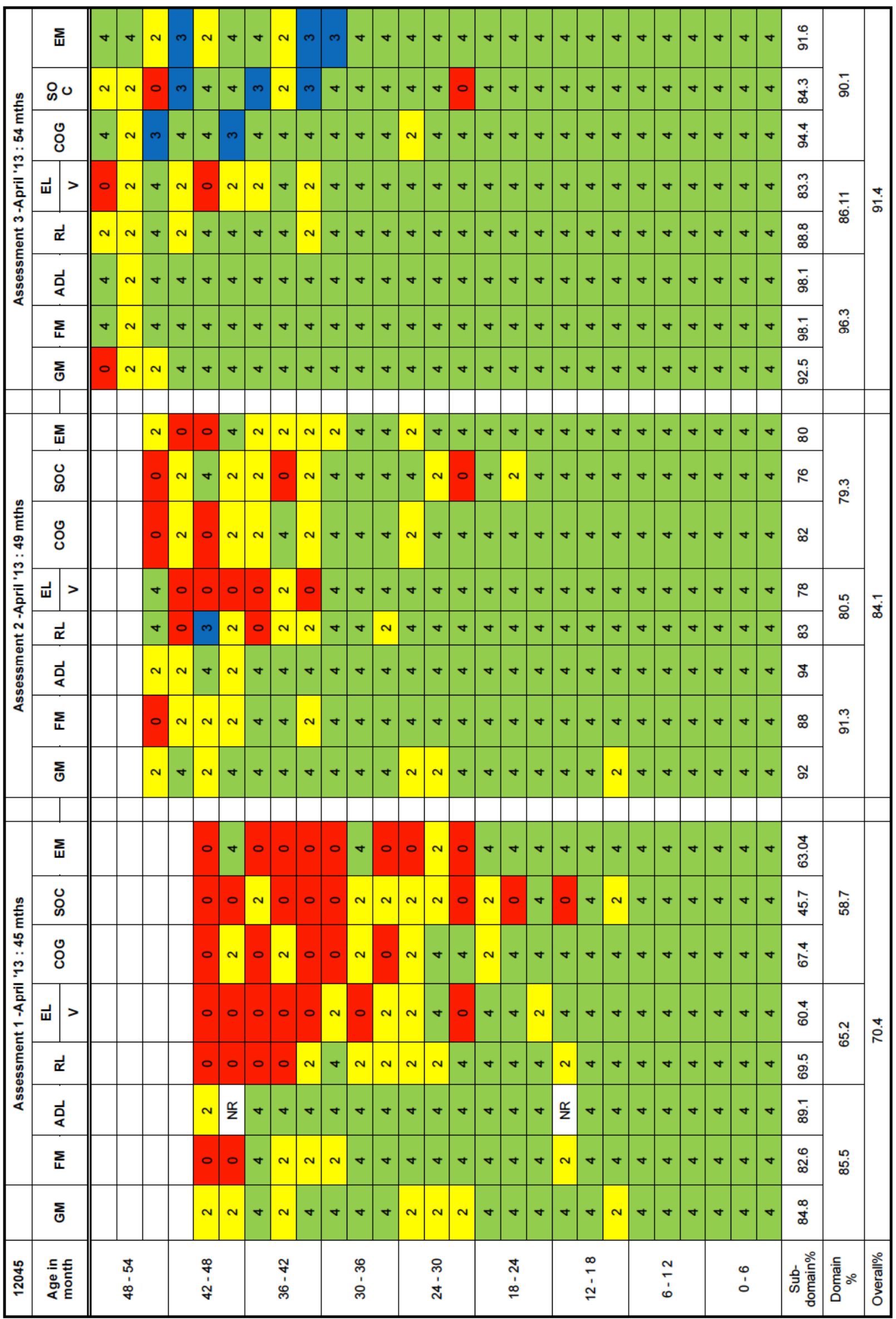


measure of skill development. For instance, while assessing a child with Hearing Impairment $(\mathrm{HI})$, the assessments specific to the hearing loss must be addressed before using the checklist. Management for a child with $\mathrm{HI}$, therefore, will be a combination of addressing the skills lacking as per the developmental checklist as well as focused on hearing management keeping in mind the need for medication, surgery, hearing aid fitting and trial. Similarly for a child with Cerebral Palsy (CP), the detailed assessment of the motor and coordination skills must be provided in addition to assessing the developmental skill levels via the checklist.

An early intervention program should take cognizance of the skill set of the child and caregiver as well as plan for inclusion in the society and school [2730]. Karanth, Shaista and Srikanth [26] explored the efficacy of the Communication DEALL program in children with Autism Spectrum Disorders (ASD). The results showed statistically significant gains across all eight developmental areas on the CDDC along with significant decrease in behavioural symptoms as measured by CARS, in the group.

\section{CONCLUSION}

An assessment that focuses on describing the strengths and deficiencies in an individual with developmental disabilities is more truly representative of the skill status of a child or individual with a developmental disability than a single measure of IQ. It not only provides directions and guidelines for intervention but can also be used to monitor the progress of the child in intervention. Unlike the IQ score it does not label and categorize the child, nor does it dictate the future course of action and prognosis on the basis of a single score. The CDDC is an example of such as an assessment for children below the age of 6 years, which also provides a baseline and guide for early broad based intervention along with provisions for monitoring and eventual placement for education.

\section{REFERENCES}

[1] Colmar S, Maxwell A, Miller L. Assessing Intellectual Disability in Children: Are IQ Measures Sufficient, or Even Necessary? Aust J Guid Couns 2006; 16(2): 177-188. http://dx.doi.org/10.1375/ajgc.16.2.177

[2] Sparrow SS, Davis SM. Recent advances in the assessment of intelligence and cognition. J Child Psychol Psychiat 2000; 41(1): 117-131.

http://dx.doi.org/10.1017/S0021963099004989

[3] Thorndike RL, Hagen EP, Sattler JM. The Stanford-Binet Intelligence Scale: Fourth edition. Technical manual. Itasca, IL: Riverside 1986.
[4] Raven J, Raven JC, Court JH. Manual for Raven's Advanced Progressive Matrices. Oxford, England: Oxford Psychologists Press 1998.

[5] Wechsler D. The WISC-IV technical and interpretive manual. San Antonio, TX: Psychological Corporation 2003.

[6] Nisbett RE, Aronson J, Blair C, Dickens W, et al. Intelligence: New findings and theoretical developments. Am Psychol 2012; 67(2): 130-159. http://dx.doi.org/10.1037/a0026699

[7] Kamin LJ. The pioneers of IQ testing. In Ressel Jacoby and Naomi Glauberman (Eds). The Bell Curve debate: History, Documents, opinions. New york: Times Book 1995.

[8] Edelson MG. Are the majority of children with autism mentally retarded? A systematic evaluation of the data. Focus Autism Other Dev Disabl 2006; 21(2): 66-83. http://dx.doi.org/10.1177/10883576060210020301

[9] Winner MG. Assessment of social skills for students with Asperger syndrome and high-functioning autism. Assess Eff Interv 2012; 27: 73-80.

http://dx.doi.org/10.1177/073724770202700110

[10] Harris SL, Handleman JS. Age and IQ at intake as predictors of placement for young children with autism: A four- to sixyear follow-up. J Autism Dev Disord 2000; 30: 137-142. http://dx.doi.org/10.1023/A:1005459606120

[11] Restori AF, Katz GS, Lee HB. A critique of the IQ/Achievment Discrepancy Model for identifying Specific Learning Disabilities. Eur J Psychol 2009; 5(4): 128-154. http://dx.doi.org/10.5964/ejop.v5i4.244

[12] Tomanik SS, Pearson DA, Loveland KA, Lane DM, Shaw JB. Improving the reliability of autism diagnoses: Examining the utility of adaptive behavior. J Autism Dev Disord 2007; 37: 921-928.

http://dx.doi.org/10.1007/s10803-006-0227-6

[13] Fenton G, D'Ardia Valente D, Vecchio ID, Fabrizi A, Bernabei $P$. Vineland adaptive behavior profiles in children with autism and moderate to severe developmental delay. Autism 2000; 7: 269-287.

http://dx.doi.org/10.1177/13623613030073004

[14] Sparrow SS, Cicchetti DV, Balla DA. Vineland-II Adaptive Behavior Scales: Survey Forms Manual. Circle Pines, MN: AGS Publishing 2005.

[15] National Research Council, Committee on Educational Interventions for Children with Autism. Educating Children With Autism. Lord C, McGee JP, editors. Washington, DC: National Academies Press 2001.

[16] Ward S. An investigation into the effectiveness of an early intervention method on delayed language development in young children. Int $\mathrm{J}$ of Lang Commun Disord 1999; 34(3): 243-264.

http://dx.doi.org/10.1080/136828299247405

[17] Landa RJ, Holman KC, O'Neill AH, Stuart EA. Intervention targeting development of socially synchronous engagement in toddlers with autism spectrum disorder: A randomized controlled trial. J Child Psychol Psychiatry 2010; 52(1): 1321. http://dx.doi.org/10.1111/j.1469-7610.2010.02288.x

[18] Matson JL, Tureck K, Turygin N, Beighley J, Rieske R. Trends and topics in Early Intensive Behavioral interventions for toddlers with autism. Res Autism Spect Dis 2012; 6(4): 1412-1417.

http://dx.doi.org/10.1016/j.rasd.2012.02.010

[19] Huttenlocher PR, Dabholkar AS. Regional differences in synaptogenesis in the human cerebral cortex. J Comp Neurol 1997; 387: 167-178.

http://dx.doi.org/10.1002/(SICl)10969861(19971020)387:2<167::AID-CNE1>3.0.CO;2-Z

[20] McEachin JJ, Smith T, Lovass OI. Long-term outcome for children with autism who received early intensive behavioral treatment. Am J Ment Retard 1993; 97(4): 359-72. 
[21] Rogers SJ, Vismara LA. Evidence-Based Comprehensive Treatments for Early Autism. J Clin Child Adolesc Psychol 2008; 37(1): 8-38. http://dx.doi.org/10.1080/15374410701817808

[22] Ozonoff S, Goodlin-Jones BL, Solomon M. Evidence-Based Assessment of Autism Spectrum Disorders in Children and Adolescents. J Clin Child Adolesc Psychol 2005; 34(3): 523540. http://dx.doi.org/10.1207/s15374424jccp3403 8

[23] Karanth P. The Communication DEALL Developmental Checklist. The Com DEALL Trust: Bangalore 2007.

[24] Karanth P. Communication DEALL- The manual. The Com DEALL Trust, Bangalore 2008.

[25] Saxena-Chandhok T, Ram-Kiran P, Lawrence L, Karanth P. The Communication DEALL Developmental Checklist - Inter rater reliability. DisabI CBR Inclu Devel 2011; 22(1): 48-54.

[26] Karanth PK, Shaista S, Srikanth N. Efficacy of communication DEALL- An indigenous early intervention program for children with Autism Spectrum disorders. Indian Journal of Pediatrics 2010; 77(9): 957-62. http://dx.doi.org/10.1007/s12098-010-0144-8
[27] Dawson G, Osterling L. Early intervention in autism. In Guralnick MJ, Ed. The effectiveness of early intervention. Baltimore: Brookes 1997; pp. 307-326.

[28] Dunlap G, Fox L. Early intervention and serious problem behaviours: a comprehensive approach. In Koegel LK, Koegel RL, et al. Eds. Positive Behvaioural support: Including people with difficult behaviours in the community. Baltimore: Paul H books publishing 1996; pp. 31-50.

[29] Calderon R, Greenberg MT. Social and emotional development of deaf children: Family, School and program effects. In Marschark M, Spencer PE, Eds. Oxford handbook of deaf studies, language and education. Oxford university press 2003; pp. 177-189.

[30] Yoshinaga-Itano C. Early intervention after universal neonatal hearing screening: Impact on outcomes. Mental Retardation and Developmental Disabilities Research Reviews 2003; 9(4): 252-266. http://dx.doi.org/10.1002/mrdd.10088

DOI: http://dx.doi.org/10.6000/2292-2598.2014.02.03.3

(C) 2014 Karanth et al.; Licensee Lifescience Global.

This is an open access article licensed under the terms of the Creative Commons Attribution Non-Commercial License (http://creativecommons.org/licenses/by-nc/3.0/) which permits unrestricted, non-commercial use, distribution and reproduction in any medium, provided the work is properly cited. 